\title{
Infrared imaging of WENSS radio sources ${ }^{\star}$
}

\author{
D. Villani ${ }^{1}$ and S. di Serego Alighieri ${ }^{2}$ \\ 1 Dipartimento di Astronomia e Scienza dello Spazio, L.go E. Fermi 5, I-50125 Firenze, Italy \\ 2 Osservatorio Astrofisico di Arcetri, L.go E. Fermi 5, I-50125 Firenze, Italy
}

Received September 17, 1997; accepted November 6, 1998

\begin{abstract}
We have performed deep imaging in the IR $J$ - and $K$ - bands for three sub-samples of radio sources extracted from the Westerbork Northern Sky Survey, a large low-frequency radio survey containing Ultra Steep Spectrum (USS), Gigahertz Peaked Spectrum (GPS) and Flat Spectrum (FS) sources. We present the results of these IR observations, carried out with the ARcetri Near Infrared CAmera (ARNICA) at the Nordic Optical Telescope (NOT), providing photometric and morphologic information on high redshift radio galaxies and quasars. We find that the radio galaxies contained in our sample do not show the pronounced radio/IR alignment claimed for $3 \mathrm{CR}$ sources. IR photometric measurements of the gravitational lens system $1600+434$ are also presented.
\end{abstract}

Key words: infrared: galaxies - radio continuum: galaxies - galaxies: photometry - gravitational lensing — atlases

\section{Introduction}

Deep near-IR imaging has an important role in the studies of very distant objects, whose redshift moves the rest frame visible light into the near-IR. In fact, from $z \geq 1$ the wavelength range where normal stars emit most of their energy and have their prominent spectral features $(4000 \leq \lambda \leq 10000 \AA)$ is shifted in the near-IR, between 1 and $2.5 \mu \mathrm{m}$. In addition, the stellar light of $z \geq 1$ galaxies observed in the near-IR is less likely to be affected by extinction and by an active nuclear component (i.e. a dust scattered AGN) than in the optical and is not contaminated by thermal dust emission. Therefore the near-IR is of fundamental importance in order to investigate the stellar component of high redshift galaxies.

Send offprint requests to: D. Villani

* This paper is based on data obtained at the Nordic Optical Telescope on La Palma (Canary Islands).

Correspondence to: villani@arcetri.astro.it
This paper describes the results of IR imaging of sources selected from the Westerbork Northern Sky Survey (WENSS, Rengelink et al. 1997), a new large-scale low-frequency radio survey that covers the whole sky for $\delta \geq 30$ degree at $325 \mathrm{MHz}$, and about a quarter of this region at $609 \mathrm{MHz}$, to a limiting flux density of 18 and 15 mJy respectively. Contrary to previous IR works concentrated mostly on powerful radio sources (e.g. Lilly \& Longair 1984; Rigler et al. 1992; Dunlop \& Peacock 1993), the radio catalogue from which we selected our sources extends to low flux densities and therefore to galaxies with a much lower level of nuclear activity.

The use of IR imaging allows one to address three important questions: first, the identification of the sources in these subsamples with their optical/IR counterparts. At high redshift $(z \geq 1)$ the peak of the spectral energy distribution of galaxies is shifted in the near IR where their flux density is therefore larger than in the optical by orders of magnitude. So it is easier to identify sources, which correspond to distant galaxies, in the IR than in the optical.

Secondly, IR imaging allows the study of the IR "alignment effect", i.e. the alignment of the IR morphology with the radio axis, as a function of the redshift and radio power. The alignment effect (see McCarthy 1993 for a review) has been discovered in the optical, and demonstrates a strong influence of the AGN on the optical morphology and luminosity. However, its origin is still matter of debate and its extension to the IR is important to understand it. Dunlop and Peacock (1993) have detected a clear IR alignment effect in a $3 \mathrm{CR}$ sample of radio galaxies in the redshift range $0.8 \leq z \leq 1.3$ (they show the IR-radio and the opticalradio alignment histograms for this subset, claiming that the alignment effect is just as clear at $K$ as in the optical) in contrast with the conclusions of Rigler et al. (1992) for the same subsample of objects. Therefore it is necessary to study the IR alignment in different samples to shed some light on the origin of this discrepancy 
Table 1. Sample of USS sources

\begin{tabular}{|c|c|c|c|c|c|c|c|c|}
\hline Object & Sub-sample & $z$ & $\begin{array}{l}\text { R.A. } \\
\text { (Epoch: J2000) }\end{array}$ & Dec. & Band & Mag. & $\begin{array}{l}\text { Apert. } \\
\text { arcsec }\end{array}$ & Notes \\
\hline $0114+331$ & $\mathrm{~B}$ & & 011651.71 & 332753.2 & $K$ & $15.99 \pm 0.24$ & 5.52 & \\
\hline \multirow[t]{2}{*}{$0114+333$} & B & & & & $K$ & $>17.73$ & 4.39 & \\
\hline & & & & & $J$ & $>19.99$ & 7.16 & \\
\hline $0114+365$ & B & & 011710.02 & 371516.4 & $K$ & $18.20 \pm 0.57$ & 3.88 & \\
\hline $0115+350$ & B & & & & $K$ & $>18.06$ & 3.28 & \\
\hline $0115+392$ & B & & 011755.07 & 394431.6 & $K$ & $17.86 \pm 0.48$ & 4.93 & \\
\hline $0123+344$ & B & & 012625.94 & 345724.9 & $K$ & $18.35 \pm 0.52$ & 3.28 & uncertain ID \\
\hline $0127+350$ & B & & 013001.54 & 352244.3 & $K$ & $16.57 \pm 0.21$ & 6.02 & uncertain ID \\
\hline $0129+353$ & B & & 013214.07 & 354816.0 & $K$ & $16.30 \pm 0.03$ & 3.83 & uncertain ID \\
\hline \multirow[t]{2}{*}{$0131+312$} & B & & 013357.99 & 314315.5 & $K$ & $18.89 \pm 0.84$ & 3.28 & \\
\hline & & & & & $J$ & $19.90 \pm 0.40$ & 3.83 & \\
\hline \multirow[t]{2}{*}{$0133+320$} & B & & 013650.44 & 322252.7 & $K$ & $>18.59$ & 3.28 & \\
\hline & & & & & $J$ & $20.61 \pm 0.31$ & 3.28 & \\
\hline $0134+323$ & B & & & & $K$ & $>17.68$ & 5.52 & \\
\hline \multirow[t]{2}{*}{$0135+311$} & B & & 013806.44 & 313241.4 & $K$ & $>18.72$ & 3.28 & \\
\hline & & & & & $J$ & $20.74 \pm 0.22$ & 3.28 & uncertain ID \\
\hline $0136+325$ & B & & 013934.05 & 331110.0 & $K$ & $17.30 \pm 0.26$ & 3.88 & uncertain ID \\
\hline \multirow[t]{2}{*}{$0136+333$} & $\mathrm{~B}$ & & 013854.23 & 335356.2 & $K$ & $16.77 \pm 0.07$ & 3.88 & uncertain ID \\
\hline & & & & & $J$ & $17.83 \pm 0.06$ & 3.88 & uncertain ID \\
\hline \multirow[t]{2}{*}{$0137+252$} & $\mathrm{C}$ & & & & $K$ & $>19.04$ & 3.28 & \\
\hline & & & & & $J$ & $>22.09$ & 3.28 & \\
\hline $0139+375$ & B & & 014207.11 & $\begin{array}{lll}38 & 05 & 46.3\end{array}$ & $K$ & $16.54 \pm 0.20$ & 4.39 & uncertain ID \\
\hline \multirow[t]{2}{*}{$0140+323$} & $\mathrm{~B}$ & & 014343.72 & 325348.3 & $K$ & $18.40 \pm 0.51$ & 3.28 & \\
\hline & & & & & $J$ & $19.73 \pm 0.12$ & 3.28 & \\
\hline \multirow[t]{2}{*}{$0143+360$} & B & & 014639.66 & 362029.1 & $K$ & $17.60 \pm 0.24$ & 3.28 & uncertain ID \\
\hline & & & & & $J$ & $20.03 \pm 0.08$ & 3.28 & uncertain ID \\
\hline \multirow[t]{3}{*}{$0148+330$} & B & & 015114.01 & 331800.7 & $K$ & $17.07 \pm 0.16$ & 3.88 & object 1 \\
\hline & & & 015113.94 & 331807.5 & $K$ & $17.51 \pm 0.24$ & 3.88 & object 2 \\
\hline & & & 015113.83 & 331811.9 & $K$ & $18.81 \pm 0.45$ & 3.88 & object 3 \\
\hline \multirow[t]{2}{*}{$0202+300$} & $\mathrm{C}$ & & 020242.65 & $\begin{array}{lll}30 & 00 & 39.3\end{array}$ & $K$ & $>18.23$ & 3.28 & \\
\hline & & & & & $J$ & $19.90 \pm 0.26$ & 3.88 & uncertain ID \\
\hline \multirow{2}{*}{$0204+392$} & B & & 020746.91 & 393754.5 & $K$ & $18.45 \pm 0.31$ & 3.88 & uncertain ID \\
\hline & & & & & $J$ & $21.11 \pm 0.67$ & 3.88 & uncertain ID \\
\hline $0214+343$ & $\mathrm{~B}$ & & 021706.00 & 344430.9 & $K$ & $17.85 \pm 0.11$ & 3.88 & \\
\hline \multirow[t]{2}{*}{$1702+604$} & $\mathrm{~A}$ & 3.23 & 170335.89 & 603848.7 & $K$ & $18.42 \pm 0.32$ & 3.88 & \\
\hline & & & & & $J$ & $>20.86$ & 3.88 & \\
\hline \multirow[t]{2}{*}{$1720+690$} & A & & 172033.98 & 690450.4 & $K$ & $17.48 \pm 0.14$ & 3.28 & \\
\hline & & & & & $J$ & $19.06 \pm 0.12$ & 3.28 & \\
\hline $1758+582$ & $\mathrm{~A}$ & 2.9 & & & $J$ & $>20.75$ & 3.28 & \\
\hline \multirow[t]{2}{*}{$1813+604$} & $\mathrm{~A}$ & & 181343.60 & 604847.3 & $K$ & $>18.24$ & 3.28 & \\
\hline & & & & & $J$ & $19.72 \pm 0.16$ & 3.28 & \\
\hline $1821+620$ & $\mathrm{~A}$ & 2.81 & 182219.56 & 620607.3 & $K$ & $16.82 \pm 0.27$ & 4.39 & \\
\hline $1842+592$ & $\mathrm{~A}$ & & 184331.37 & 593257.8 & $K$ & $17.96 \pm 0.19$ & 3.28 & \\
\hline $1842+691$ & $\mathrm{~A}$ & & & & $K$ & $>17.88$ & 3.28 & \\
\hline $1854+622$ & $\mathrm{~A}$ & & & & $K$ & $>18.56$ & 3.28 & \\
\hline $1913+672$ & $\mathrm{~A}$ & & 191315.56 & 673222.0 & $K$ & $17.01 \pm 0.36$ & 3.88 & uncertain ID \\
\hline $2320+122$ & $\mathrm{C}$ & & 232007.73 & 122205.4 & $K$ & $18.45 \pm 1.36$ & 3.28 & \\
\hline $2321+223$ & $\mathrm{C}$ & & 232142.36 & 223756.8 & $K$ & $17.67 \pm 0.78$ & 3.88 & uncertain ID \\
\hline $2334+154$ & $\mathrm{C}$ & & 233458.49 & 154551.9 & $K$ & $17.78 \pm 0.35$ & 4.39 & uncertain ID \\
\hline $2334+313$ & $\mathrm{C}$ & & 233415.16 & 313930.9 & $K$ & $18.22 \pm 0.24$ & 3.28 & uncertain ID \\
\hline \multirow[t]{4}{*}{$2351+103$} & $\mathrm{C}$ & & 235126.79 & 103456.2 & $K$ & $17.35 \pm 0.61$ & 4.39 & east component \\
\hline & & & 235126.57 & 103455.7 & $K$ & $17.82 \pm 0.87$ & 4.39 & west component \\
\hline & & & & & $J$ & $19.08 \pm 0.08$ & 4.39 & east component \\
\hline & & & & & $J$ & $19.80 \pm 0.15$ & 4.39 & west component \\
\hline
\end{tabular}


Table 2. Sample of GPS sources

\begin{tabular}{|c|c|c|c|c|c|c|c|c|}
\hline Object & $z$ & $R$ & Type $^{*}$ & $\begin{array}{l}\text { R.A. } \\
\text { (Epoch: J2000) }\end{array}$ & Dec. & Band & Mag. & $\begin{array}{l}\text { Apert. } \\
\text { arcsec }\end{array}$ \\
\hline $1620+640$ & & 23.58 & $\mathrm{G}$ & 162115.22 & 635914.1 & $K$ & $18.62 \pm 0.41$ & 3.28 \\
\hline $1622+663$ & 0.20 & 17.15 & $\mathrm{G}$ & 162304.38 & 662401.4 & $K$ & $13.24 \pm 0.04$ & 7.70 \\
\hline $1642+670$ & 1.91 & 17.03 & Q & 164221.67 & 665548.6 & $K$ & $15.05 \pm 0.03$ & 4.39 \\
\hline $1647+622$ & 2.19 & 20.41 & Q & & & $K$ & $>17.92$ & 3.88 \\
\hline \multirow[t]{2}{*}{$1657+582$} & & 23.30 & FG & 165805.05 & 582202.4 & $K$ & $17.80 \pm 0.11$ & 3.28 \\
\hline & & & & & & $J$ & $19.40 \pm 0.15$ & 3.28 \\
\hline $1746+692$ & 1.88 & 19.22 & Q & 174629.92 & $\begin{array}{lll}69 & 2035.7\end{array}$ & $K$ & $16.51 \pm 0.12$ & 3.88 \\
\hline \multirow[t]{2}{*}{$1808+681$} & & 23.27 & FG & $18 \quad 08 \quad 12.24$ & $6814 \quad 11.0$ & $K$ & $>18.85$ & 3.28 \\
\hline & & & & & & $J$ & $20.11 \pm 0.28$ & 3.28 \\
\hline \multirow[t]{2}{*}{$1819+670$} & 0.22 & 17.72 & $\mathrm{G}$ & 181944.22 & 670846.4 & $K$ & $14.44 \pm 0.07$ & 6.60 \\
\hline & & & & & & $J$ & $15.85 \pm 0.03$ & 6.60 \\
\hline $1841+671$ & 0.47 & 20.53 & $\mathrm{G}$ & 184103.68 & 671850.2 & $K$ & $16.80 \pm 0.54$ & 4.39 \\
\hline $1942+721$ & & 23.00 & $\mathrm{G}$ & 194126.69 & 722142.3 & $J$ & $20.36 \pm 0.17$ & 3.28 \\
\hline \multirow[t]{2}{*}{$1945+602$} & 2.70 & 20.42 & $\mathrm{Q}$ & $1946 \quad 13.14$ & 603138.0 & $K$ & $15.65 \pm 0.06$ & 3.28 \\
\hline & & & & & & $J$ & $17.49 \pm 0.05$ & 3.28 \\
\hline \multirow[t]{4}{*}{$1946+704$} & 0.101 & 16.33 & $\mathrm{G}$ & 194553.40 & 705548.9 & $K$ & $13.81 \pm 0.07$ & 7.68 \\
\hline & & & & & & $K$ & $13.42 \pm 0.30$ & $23.66^{* *}$ \\
\hline & & & & & & $J$ & $14.80 \pm 0.04$ & 7.68 \\
\hline & & & & & & $J$ & $14.23 \pm 0.24$ & $23.66^{* *}$ \\
\hline \multirow[t]{2}{*}{$1954+614$} & & 22.18 & $\mathrm{G}$ & 195456.16 & 615357.1 & $K$ & $16.31 \pm 0.07$ & 3.28 \\
\hline & & & & & & $J$ & $18.11 \pm 0.05$ & 3.28 \\
\hline \multirow[t]{2}{*}{$1958+615$} & 1.81 & 22.40 & Q & 195930.07 & 620645.1 & $K$ & $18.07 \pm 0.15$ & 3.28 \\
\hline & & & & & & $J$ & $19.57 \pm 0.18$ & 3.28 \\
\hline
\end{tabular}

${ }^{*} \mathrm{G}=$ galaxy; $\mathrm{FG}=$ faint galaxy; $\mathrm{Q}=$ quasar.

**These apertures were used for comparison with optical photometry (Snellen, PhD thesis).

Table 3. Sample of FS sources

\begin{tabular}{|c|c|c|c|c|c|c|c|c|}
\hline Object & $z$ & $R$ & $\begin{array}{l}\text { R.A. } \\
\text { (Epoch: J2000) }\end{array}$ & Dec. & Band & Mag. & $\begin{array}{l}\text { Apert. } \\
\text { arcsec }\end{array}$ & Notes \\
\hline $1701+552$ & & $>22.50$ & & & $K$ & $>18.24$ & 3.28 & \\
\hline \multirow[t]{2}{*}{$1702+637$} & & $>22.50$ & 170304.73 & 634233.8 & $K$ & $18.44 \pm 0.50$ & 3.28 & uncertain ID \\
\hline & & & & & $J$ & $19.86 \pm 0.19$ & 3.88 & uncertain ID \\
\hline $1712+651$ & & $>22.50$ & & & $K$ & $>18.21$ & 3.28 & \\
\hline $1744+678$ & 3.40 & 18.50 & 174442.10 & 675046.5 & $K$ & $16.19 \pm 0.08$ & 4.39 & \\
\hline $1754+676$ & 3.60 & 19.80 & 175422.14 & 673735.9 & $K$ & $17.74 \pm 0.43$ & 3.28 & \\
\hline $1807+599$ & & $>22.50$ & 180755.56 & 600000.2 & $K$ & $17.99 \pm 0.49$ & 4.93 & \\
\hline $1816+615$ & & $>22.50$ & 181655.27 & 613444.4 & $K$ & $18.39 \pm 0.17$ & 3.28 & \\
\hline $1841+568$ & & 19.25 & 184243.90 & 565640.3 & $K$ & $16.53 \pm 0.20$ & 3.88 & uncertain ID \\
\hline $1846+610$ & & $>22.50$ & 184724.45 & 610750.2 & $K$ & $17.59 \pm 0.44$ & 3.88 & \\
\hline $1849+643$ & & 14.50 & 184940.90 & 642515.1 & $K$ & $12.12 \pm 0.04$ & 20.35 & bright galaxy \\
\hline $1851+608$ & & 21.00 & 185232.06 & 605313.8 & $K$ & $16.97 \pm 0.42$ & 4.93 & \\
\hline $1854+605$ & & 21.00 & 185442.24 & 603458.2 & $K$ & $17.27 \pm 0.35$ & 3.88 & \\
\hline $1857+710$ & & 22.00 & & & $K$ & $>17.08$ & 4.39 & \\
\hline
\end{tabular}

(probably, much of the apparent discrepancy arises from different methods of analysis) and on the possible presence of different components which dominate in the IR and in the optical, respectively.

Finally, the IR observations enable the study of the stellar populations of distant galaxies through the analysis of the light emitted by normal stars, in order to determine, modelling their spectral energy distributions by means of stellar population synthesis models, the age of the oldest stars and therefore provide us with informa- tion about the epoch of galaxy formation. We emphasize that the starlight analysis is easier and more productive in the samples at lower radio power, as are the ones selected from the WENSS survey, since the properties of the stellar populations are less contaminated by the AGN component.

In the next paragraph we discuss the selection criteria of our samples. Then we report on our observations and immediate results for individual sources. We then analyse 
Table 4. Position angles in the USS sources. For those galaxies indicated by a question mark in Col. 2, we have only one radio component. The numbers with colon are uncertain

\begin{tabular}{llll}
\hline Object & $\begin{array}{l}\text { Radio PA } \\
\left({ }^{\circ}\right)\end{array}$ & $\begin{array}{l}\text { IR PA } \\
\left({ }^{\circ}\right)\end{array}$ & $\begin{array}{l}\Delta \text { PA (Radio-IR) } \\
\left({ }^{\circ}\right)\end{array}$ \\
\hline $0114+331$ & no radio map & round image \\
$0114+365$ & $?$ & $65:$ & \\
$0115+392$ & 167 & 110 & 57 \\
$0131+312$ & $127:$ & $90:$ & $37:$ \\
$0133+320$ & 150 & too faint & \\
$0140+323$ & 104 & $80:(\mathrm{J})$ & $24:$ \\
$0204+392$ & 26 & too faint & \\
$0214+343$ & $73:$ & $140:$ & $67:$ \\
$1702+604$ & 176 & too faint & \\
$1720+690$ & $?$ & round image & \\
$1813+604$ & $?$ & round image & \\
$1821+620$ & $?$ & 102 & \\
$1842+592$ & 76 & 13 & 63 \\
$2320+122$ & no radio map & too faint \\
$2351+103$ & no radio map & 80 & \\
\hline
\end{tabular}

Table 5. Position angles in the GPS sources. The numbers with colon are uncertain

\begin{tabular}{llll}
\hline Object & $\begin{array}{l}\text { Radio PA } \\
\left({ }^{\circ}\right)\end{array}$ & $\begin{array}{l}\text { IR PA } \\
\left({ }^{\circ}\right)\end{array}$ & $\begin{array}{l}\Delta \text { PA (Radio-IR) } \\
\left({ }^{\circ}\right)\end{array}$ \\
\hline $1620+640$ & 169 & round image \\
$1622+663$ & 61 & 111 & 50 \\
$1657+582$ & 30 & 170 & 40 \\
$1808+681$ & 172 & too faint & \\
$1819+670$ & 44 & 160 & 64 \\
$1841+671$ & 169 & $50:$ & $61:$ \\
$1942+721$ & 62 & 70 & 8 \\
$1946+704$ & 27 & round image & \\
$1954+614$ & 150 & round image \\
\hline
\end{tabular}

Table 6. Observing parameters of the gravitational lens system $1600+434$

\begin{tabular}{lllll}
\hline Observing date & $\begin{array}{l}\text { Obs. time } \\
\text { min. }\end{array}$ & Band & Mag. ${ }^{*}$ & $\begin{array}{l}\text { Apert. } \\
\text { arcsec }\end{array}$ \\
\hline 14 Aug. 1995 & 30 & $K$ & $16.65 \pm 0.12$ & 4.39 \\
16 Aug. 1995 & 30 & $J$ & $19.78 \pm 0.12$ & 4.39 \\
\hline
\end{tabular}

${ }^{*}$ Lensing galaxy only.

the radio-IR alignment and discuss the results obtained for a gravitational lens system.

\section{Selection criteria}

Our work is part of a large collaboration for optical and infrared studies of radio sources from the WENSS catalogue. For this purpose, three samples of the WENSS catalogue have been defined:

1. Ultra Steep Spectrum (USS) radio sources with a flux density $100<S_{325 \mathrm{MHz}}<500 \mathrm{mJy}$ and a steep spectral index cut-off of $\alpha_{609}^{325}<-1.1\left(S \propto \nu^{\alpha}\right)$. Optical and infrared follow-up work has been limited to the 95 per cent of this sample which has no identification on POSS. These objects are powerful tracers of high redshift galaxies.

2. Gigahertz Peaked Spectrum (GPS) radio sources located in two regions of the survey: one at $15^{\mathrm{h}}<$ R.A. $<20^{\mathrm{h}}$ and $58^{\circ}<$ dec. $<75^{\circ}$, which is called the mini-survey region (Rengelink et al. 1997), centered on the North Ecliptic Pole, and the other at $4^{\mathrm{h}} 00^{\mathrm{m}}<$ R.A. $<8^{\mathrm{h}} 30^{\mathrm{m}}$ and $58^{\mathrm{o}}<$ dec. $<75^{\circ}$. These sources have a spectral index cut-off of $\alpha_{609}^{325}>0.0$ with a convex radio spectrum peaked at a frequency of about $1 \mathrm{GHz}$ (Snellen et al. 1998). They are compact luminous objects, at intermediate and high redshift, which are interesting both as a special class of AGN and as probes of galaxy evolution.

3. Flat Spectrum (FS) radio sources with an initial selection from the Greenbank Surveys (Condon \& Broderick 1985; Gregory \& Condon 1991) at $5 \mathrm{GHz}$ $\left(S_{5 \mathrm{GHz}}>20 \mathrm{mJy}\right)$. The coordinate limits are $17^{\mathrm{h}}<$ R.A. $<19^{\mathrm{h}}$ and dec. $>55^{\mathrm{o}}$ and the spectral index cut-off $\alpha_{609}^{325}>-0.5$. They are mostly quasars, up to the highest redshifts, along with a useful number of radio-faint $\mathrm{BL}$ Lacs.

The sub-samples of sources to be observed in the IR were defined from the above samples in the following way:

1. USS sources:

(a) Sub-sample A with $16^{\mathrm{h}}<$ R.A. $<19^{\mathrm{h}} 15^{\mathrm{m}}$ and dec. $>55^{\circ}$ and spectral index $\alpha_{5000}^{327}<-1.2$. At the time of selection optical $R$-band images and spectra were already available.

(b) Sub-sample B with $1^{\mathrm{h}}<$ R.A. $<2^{\mathrm{h}} 15^{\mathrm{m}}$ and $30^{\mathrm{o}}<$ dec. $<40^{\circ}$. For these objects we had also $R$-band images and VLA maps.

(c) Sub-sample C with $23^{\mathrm{h}}<$ R.A. $<2^{\mathrm{h}}$. It is a high flux control sample supplied by Richard Saunders. It was selected to have a flux density larger than $0.9 \mathrm{Jy}$ at $365 \mathrm{MHz}$, to be about a factor $5-10$ brighter in the radio than the sources in the rest of the sample. For these objects we had only VLA maps.

Given they overlap in right ascension range with the GPS and FS sources, we observed in the IR only 9 out of the 69 objects in sub-sample A. On the other hand, we observed most objects of subsample B (20 out of 30 ) and all 7 objects of subsample C.

2. GPS sources in the mini-survey region further constrained to R.A. $>16^{\mathrm{h}}$, with optical identification in the $R$-band. We have imaged in $K$-band all the 14 objects in this sub-sample. 8 of them were also imaged in the $J$-band.

3. No further selection was applied a priori on the FS sample. However given the available observing time, we imaged in $K$-band 13 out of the 67 sources in the 

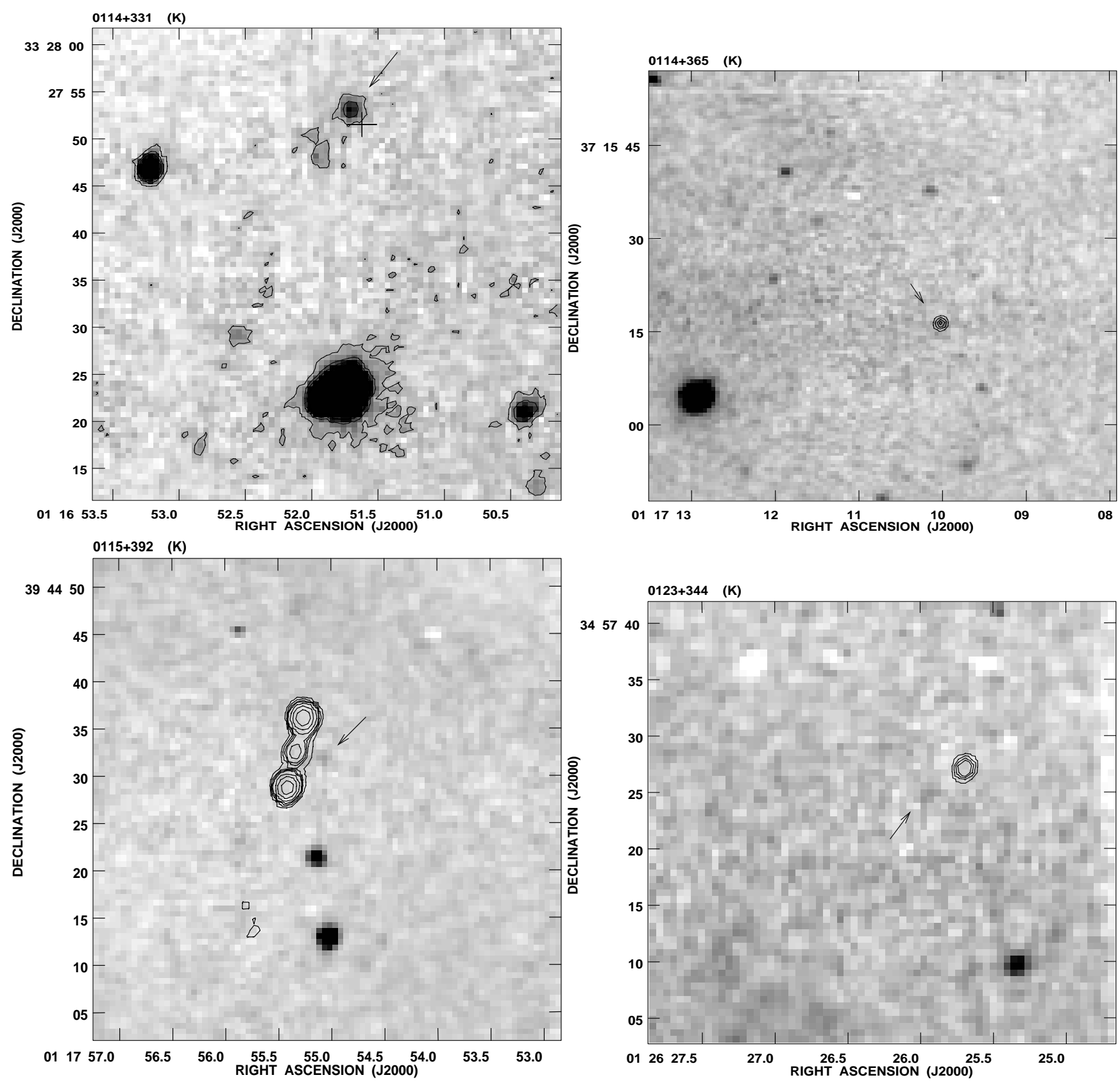

Fig. 1. $K$ - and $J$-band images (grey scale) of USS, GPS and FS radio sources. The arrows point to the IR counterparts. The crosses indicate the VLA positions of the radio cores or of the radio centroids; when available, the VLA radio contours are superimposed on the IR images. In the case of $0114+331$ we show also contour levels of the IR flux, in order to enhance the two companions of the source at 4.5 arcsec to the south-east. In a few cases, bad pixels or columns are present, although they were corrected for the photometry

FS sample. These were chosen to cover the full range of flux densities at $5 \mathrm{GHz}$ in the sample and to contain a fair number (5) of sources not identified on deep CCD frames in the $R$-band.

\section{Observations and reduction}

The observations were performed in two runs (August and September 1995), using the Nordic Optical Telescope
(NOT) at La Palma, equipped with the ARcetri NearInfrared CAmera (ARNICA, Lisi et al. 1994) and they were part of the 1995 International Time Programme for the telescopes on the Canary Islands on optical/IR followup studies of WENSS sources.

ARNICA relies on a $256 \times 256 \mathrm{HgCdTe}$ NICMOS array and was used with a scale of $0.55 \mathrm{arcsec} / \mathrm{pixel}$ and a field of view of 2.35 arcmin.

Tables 1, 2, 3 list the observed sources for the USS, GPS, and FS samples respectively. In the first columns of 

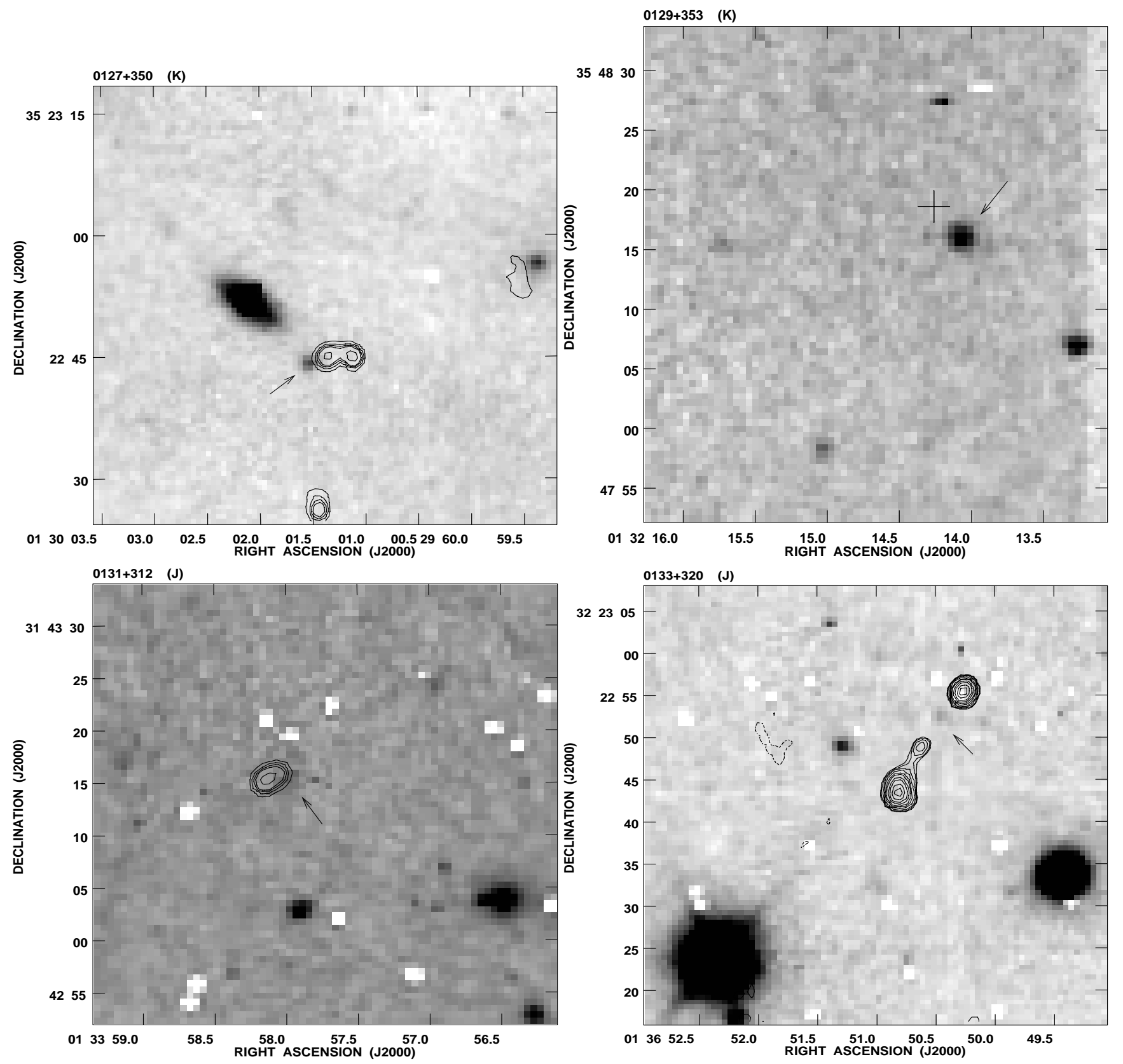

Fig. 1. continued

the Tables we give some information from other authors on the optical identifications of the three samples.

All sources were observed in $K$-band. Some of USS and GPS sources, in particular those which were not clearly detected on the $K$-band images after the quicklook reduction at the telescope, were also observed in the $J$-band to increase the chances of an infrared detection. We used the method developed by Tyson (1986) and collaborators for deep CCD imaging, in order to ensure background-limited images and avoid the saturation of the brightest sources. To this aim, for each object, the total exposure time was broken up into a number of shorter exposures, background-limited, between which the telescope was moved in a 9 position raster. For calibration purposes

we observed standard stars, typically four per night, from the ARNICA list (Hunt et al. 1998). The seeing was good throughout our runs, between 1.0 and 0.5 arcsec and conditions were mostly photometric.

The reduction of the images employed the IRAF procedures available in the ARNICA data reduction package. In order to eliminate the spatial variations in the detector response, arising from camera vignetting and variation in the quantum efficiency of the detector, we applied the flat field correction, that is the division into each frame of a sky flat frame. As a rule, for each of the 9 positions of the telescope, the data reduction procedures compute a weighted median of the images of the other 8 positions (or a subset of them) to form the sky frame for flat-fielding. 

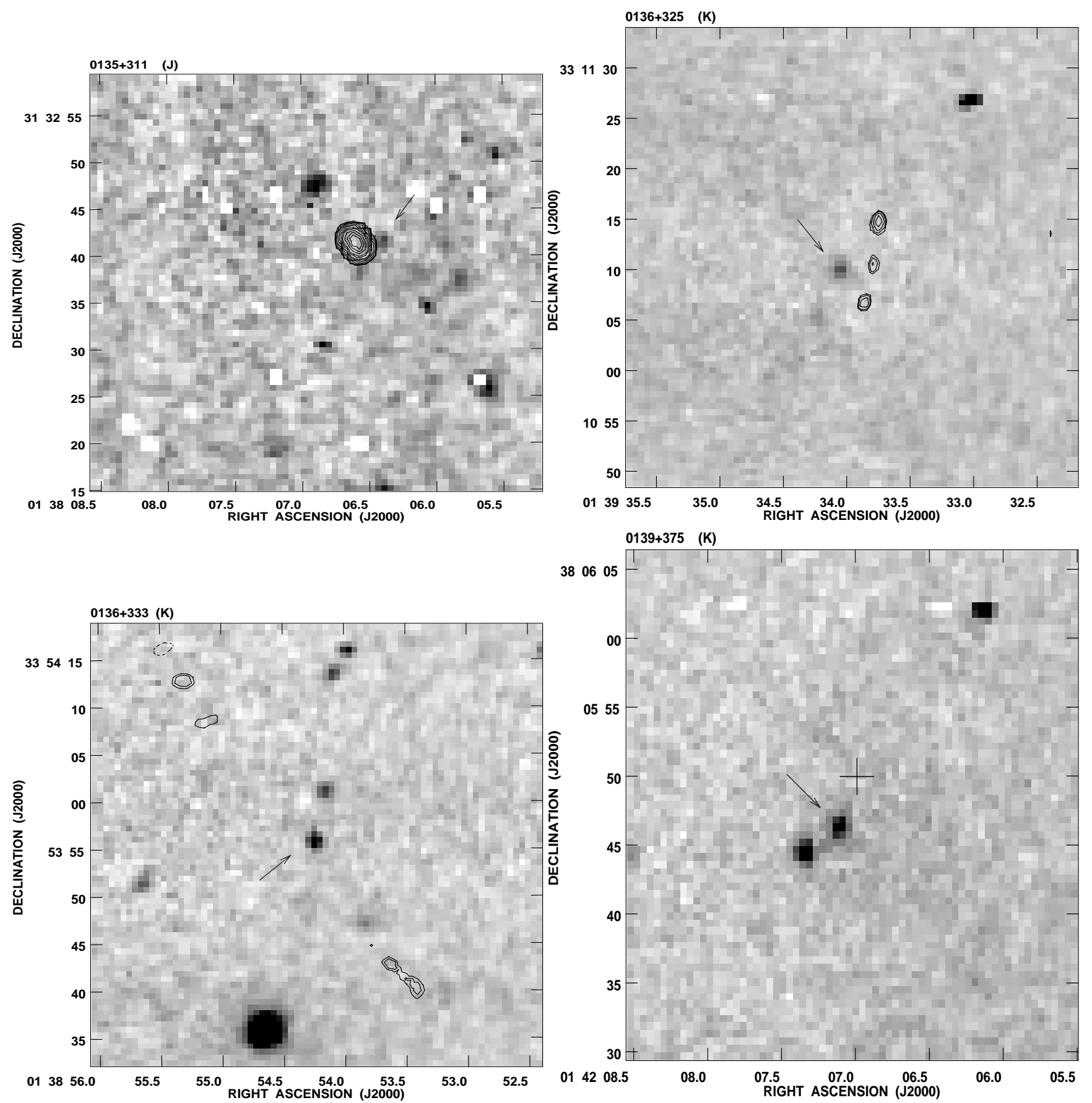

Fig. 1. continued

However, in the case of $K$-band images, it was necessary to do the background subtraction from each frame before flat-fielding by means of normalized differential sky flats (Hunt et al. 1994) in order to eliminate the contribution of the thermal emission from the telescope that becomes significant for this band. Finally, the frames were combined together to produce the final source frame, using a new algorithm, invented by Hook \& Fruchter (1997) in order to combine multiple stacks of dithered, undersampled image frames.

\section{IR identifications and photometry}

For the identification of the IR counterparts of radio sources we relied on the following informations: the most accurate radio positions available and the optical positions of stars visible in our IR frames. Concerning the radio positions, for the sources observed at the VLA we used the VLA coordinates of the radio core. In case the radio core was not detected we used the WENSS positions of the radio sources and we found it useful to overlay the VLA radio maps, when available, on our IR frames. 

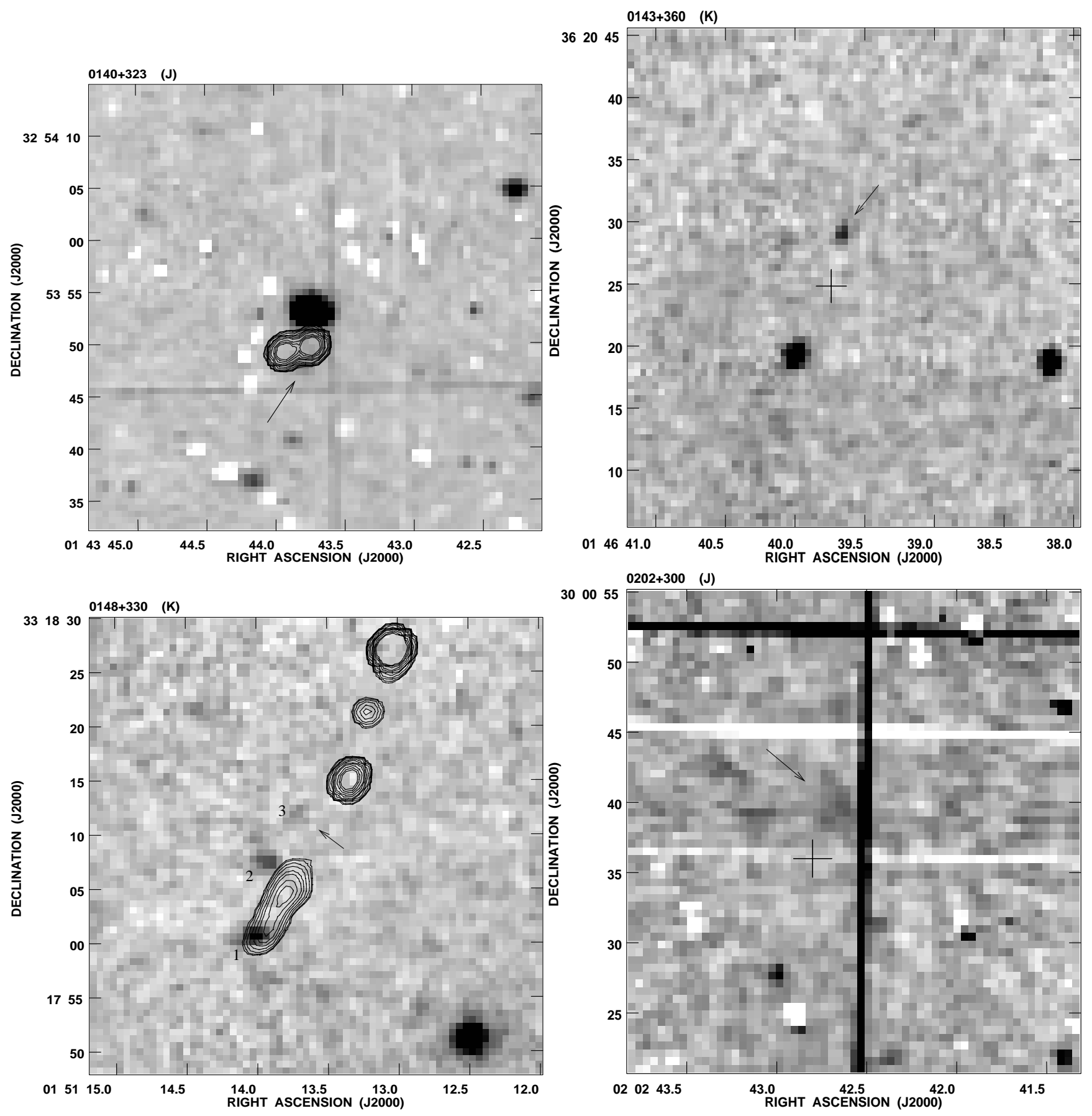

Fig. 1. continued

For positioning the radio structure over our IR images, we needed astrometric information for our IR images. This was obtained using stellar objects in common between our images and $R$-band CCD images for which the astrometric work was already done (Rengelink \& Snellen, private communication), or, when these were not available, using optical sky maps extracted from the digitized sky survey (DSS) plates available at the Space Telescope Science Institute.

The accuracy of our astrometric work depends on several factors: the uncertainties in the optical right ascen-

sion and declination positions; the errors in the radio positions; the accuracy in the radio/IR overlay procedure. The accuracy of the optical positions from the DSS plates is about 1 to 2 arcsec, depending on the distance of the object from the center of the Schmidt plate. The positional errors in the VLA radio maps are about 1 arcsec or better. The WENSS radio coordinates (used for some USS sources lacking VLA radio maps) have a 1 sigma error on the position better than 5 arcsec. For double sources that do not show an obvious radio core component, the extent of the radio source introduces an additional 

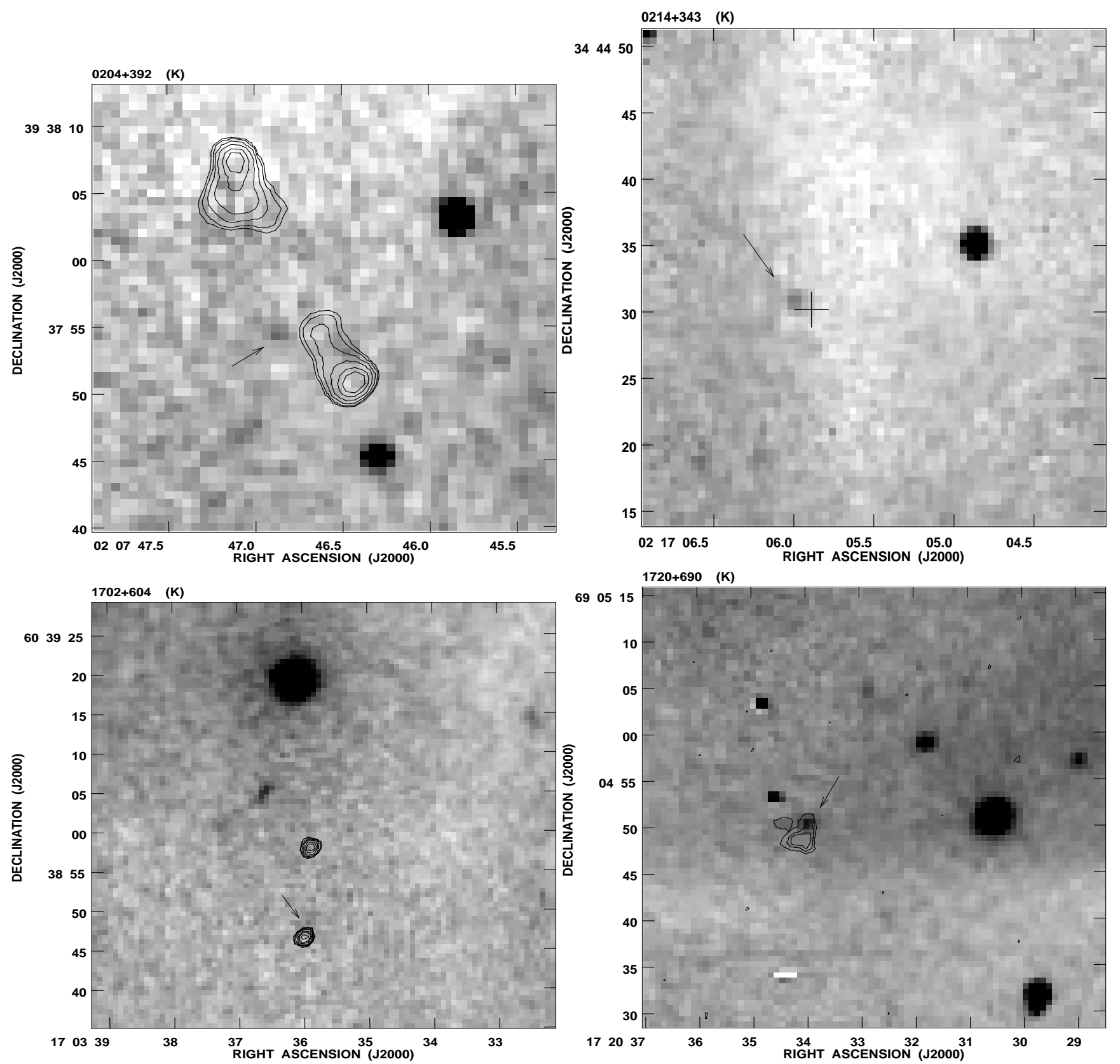

Fig. 1. continued

indetermination in the position of the IR counterpart. Finally, the accuracy of our radio/IR overlay procedure is about 0.5 arcsec. Taking into account all these uncertainties, we have accepted as good identifications those for which the distance between the radio and IR position is less than 1.5 to 5 arcsec, depending on the astrometric information available.

Tables 1, 2, 3 list the coordinates of the IR counterparts. In many cases, our IR detections provided us with an useful tool in order to confirm the identification of the fainter optical sources and to find the counterparts of the sources still lacking optical identifications. Figure 1 shows the $K$ - and $J$-band images of the sources for which we have an IR counterpart, even if uncertain.

For all the IR counterparts, we measured the magnitudes using circular apertures with the minimum diameter which includes all the detected object flux. We tested this procedure by making several measurements with different aperture diameters. In a few cases we adjusted the photometric aperture to correspond to that used for the same object on the optical image by Snellen. The size of the apertures and the measured magnitudes, corrected for galactic foreground extinction, are listed in Tables 1, 2 and 3 for the three samples respectively. The photometric accuracy given in Tables 1, 2, 3 has been evaluated from the measured fluctuations of the sky background around the source for measurement apertures equivalent to that used for source itself. If no IR counterpart is detected we give 

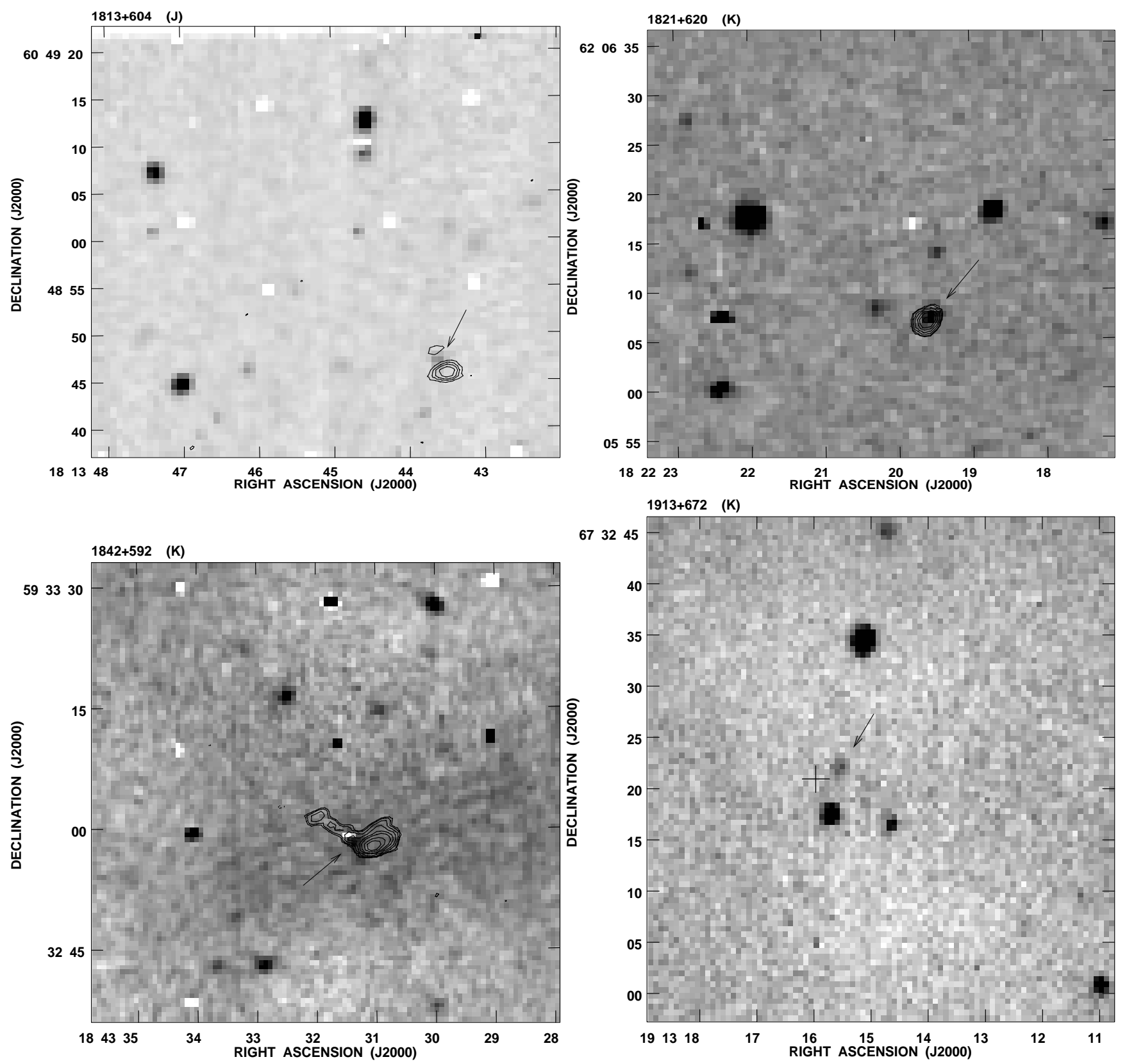

Fig. 1. continued

$3 \sigma$ upper limit based on the background fluctuations. The $J-K$ colours which we obtain for the USS sources and for the galaxies in the GPS sample are within the range of those observed for distant radio galaxies (e.g. Lilly \& Longair 1984).

\section{Comments on individual objects}

\subsection{Sample of USS sources}

$0114+331$. The object is resolved but round in $K$-band and has two companions at 4.5 arcsec to the south-east which are not included in our photometry. $\mathbf{0 1 1 5}+\mathbf{3 9 2}$. The IR counterpart is at 3 arcsec to the west of the central radio component.

$\mathbf{0 1 2 3 + 3 4 4}$. We give as a tentative identification a faint object at 4 arcsec to the south-east of the radio source which is visible also in the $R$-band.

$\mathbf{0 1 2 7}+\mathbf{3 5 0}$. We give as a tentative identification a compact object near the eastern component of the northern radio lobe.

$\mathbf{0 1 2 9}+\mathbf{3 5 3}$. Our identification is at 3.6 arcsec to the southwest of the radio position.

$\mathbf{0 1 3 3 + 3 2 0}$. The faint object visible in $J$ but not in $K$ corresponds to the optical identification (Rengelink, private communication). 

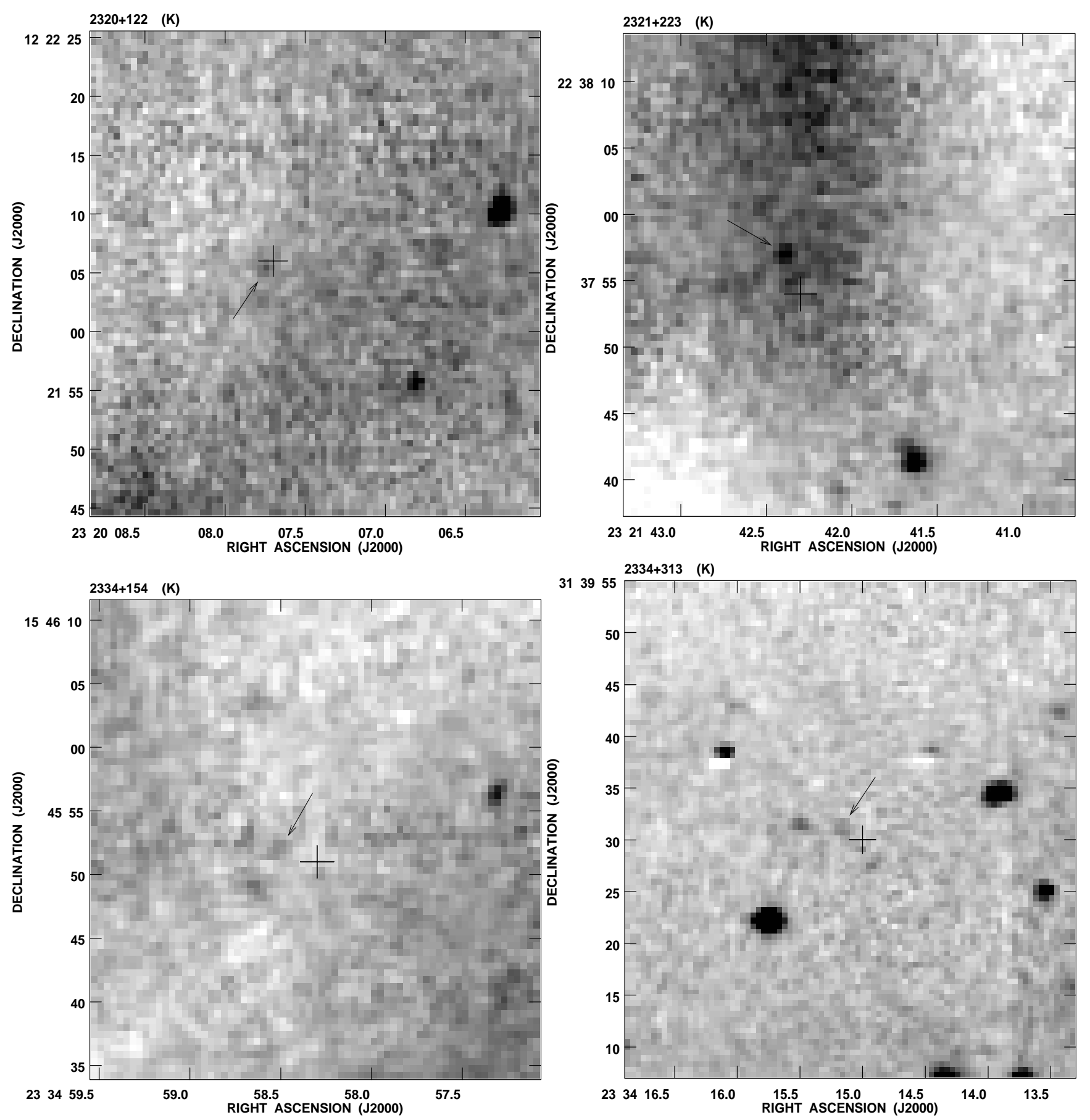

Fig. 1. continued

$\mathbf{0 1 3 5}+\mathbf{3 1 1}$. Our tentative identification corresponds to a faint object in the $R$-band.

$\mathbf{0 1 3 6}+\mathbf{3 2 5}$. We give the magnitude of the object at 3 arcsec to the east of the central radio component. It is visible also in the $R$-band.

$\mathbf{0 1 3 6}+\mathbf{3 3 3}$. We have measured the object which is at about half way between the two radio lobes.

$\mathbf{0 1 3 9 + 3 7 5}$. We give the magnitude of the object at 4 arcsec to the south of the radio position.

$\mathbf{0 1 4 0 + 3 2 3}$. Our identification is at 6 arcsec to the south of a brighter galaxy.

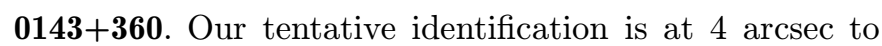
the north of the radio position.

$\mathbf{0 1 4 8 + 3 3 0}$. We have measured the three object shown in the figure. The most likely but still uncertain identification is the object number 3 .

$\mathbf{0 2 0 2 + 3 0 0}$. We give as a tentative identification the extended object visible only in $J$ at 3 arcsec to the northwest of the radio position. 

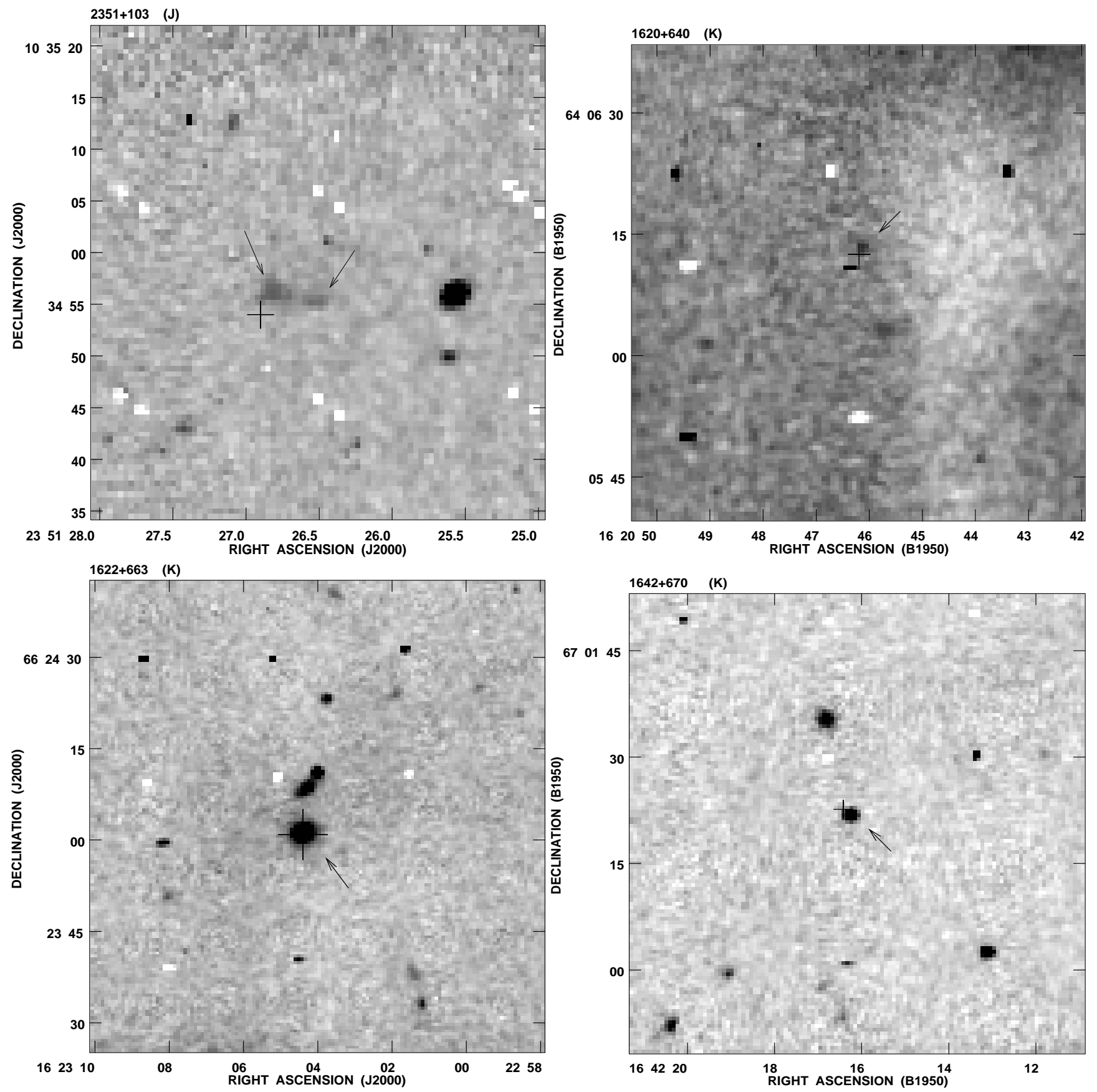

Fig. 1. continued

1702+604. The identification is just to the north of the southern radio lobe and corresponds to the galaxy visible in the $R$-band, for which the redshift has been measured (Bremer, private communication).

1913+672. Our tentative identification is $2.5 \operatorname{arcsec}$ to the north-west of the radio position.

2321+223. Our tentative identification is at 3 arcsec to the north of the radio position.

$\mathbf{2 3 3 4}+\mathbf{1 5 4}$. Of the 3 objects to the east of the radio position, we give as a tentative identification the closest one.
2334+313. Our tentative identification is the central object of the 3 around the radio position.

2351+103. The object has two components in both $J$ - and $K$-bands which we measured separately.

\subsection{Sample of GPS sources}

$\mathbf{1 9 5 8 + 6 1 5}$. The quasar is at 3 arcsec to the north-west of a star (Snellen, PhD thesis). 

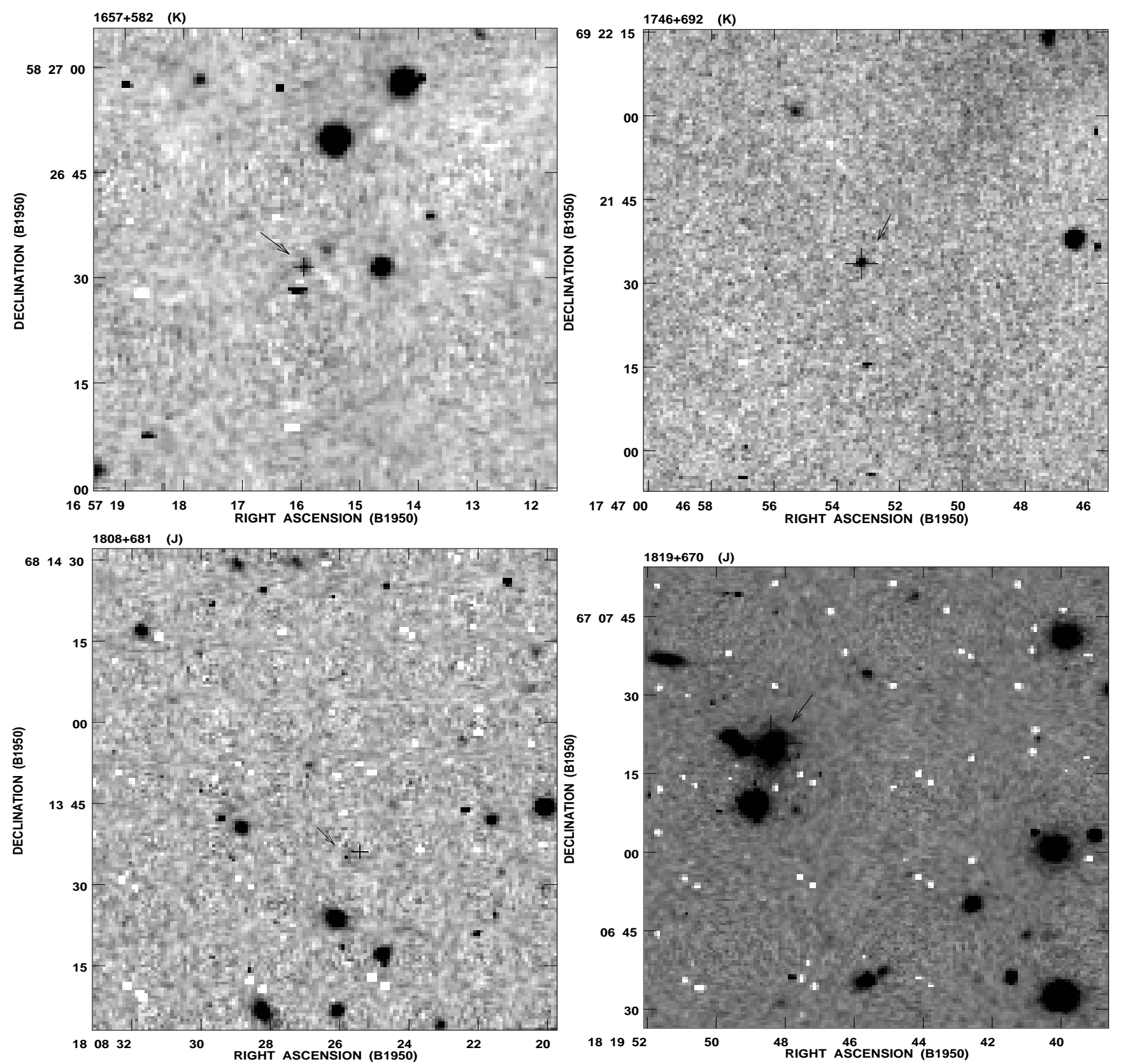

Fig. 1. continued

\subsection{Sample of FS sources}

1702+637. Our identification is at 4 arcsec to the north of the radio position.

$\mathbf{1 8 4 1}+\mathbf{5 6 8}$. Our identification is at 3 arcsec to the south of the radio position.

$\mathbf{1 8 5 4 + 6 0 5}$. Our tentative identification is 3.6 arcsec to the south-west of the radio position.

\section{IR-radio alignment effect}

We give in Tables 4 and 5 the radio and IR position angles along with the differences between them for the USS

and GPS subsamples of radio galaxies. In case of USS sources we used the VLA radio maps provided us from Rengelink (private communication) in order to measure the radio position angles. After selecting the objects with definite identification, we have not found evidence for an IR alignment effect: 3 objects, although resolved, show an IR round morphology; 4 objects are too faint in the IR to say if they are elongated; for 5 objects we have measured radio to IR position angle differences clearly not biased toward zero.

These results, although obtained on a small number of objects, suggest that the IR alignment in our sample of USS sources is not as strong as detected in the optical on powerful distant radio sources (e.g. McCarthy 1993). 

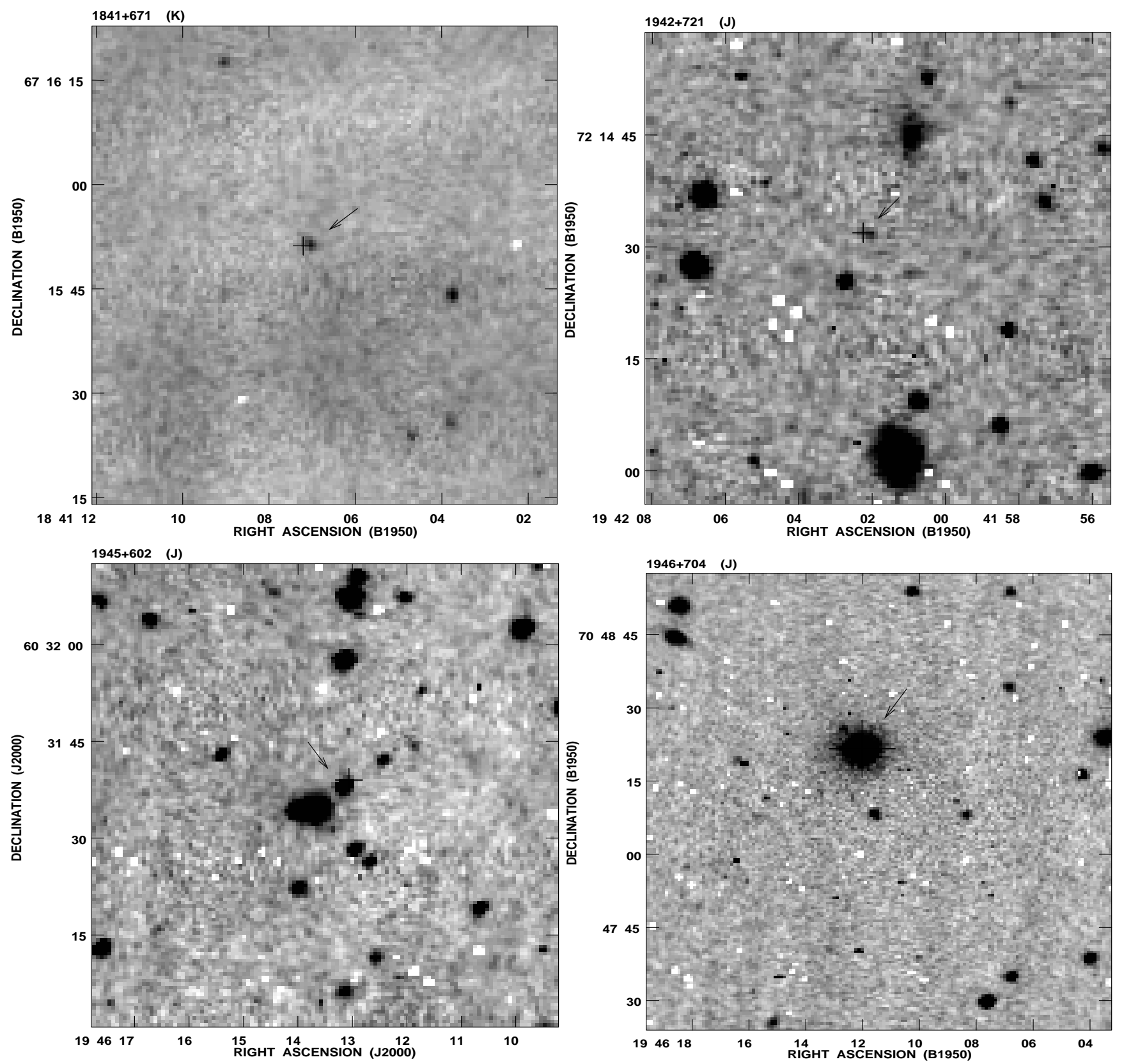

Fig. 1. continued

Furthermore, we are unable to confirm on our sample the claim for a precise IR-radio alignment obtained by Dunlop $\&$ Peacock (1993) for 3CR sources at $z \sim 1$. We suggest that the lack of alignment in our sample may be connected with its lower radio power. It seems in fact plausible that the alignment effect, if it is a manifestation of the nuclear activity on the optical/IR morphology, would decrease with radio power which is a measure of nuclear activity.

In the case of GPS sources we have defined the radio position angles using the analysis of multifrequency VLBI observations carried out by Snellen ( $\mathrm{PhD}$ thesis) for our sample of faint objects. In all cases, we chose the lowest frequency maps to enhance the importance of the extended parts. The IR position angles of GPS galaxies are in general not aligned with the radio axis (see Table 5). The only exception $(1942+721)$ could well happen by chance. Furthermore, 3 objects show an IR round morphology and 1 object is too faint in the IR to measure any orientation. The lack of optical-radio alignment in GPS sources has also been noticed by Snellen et al. (1996). 

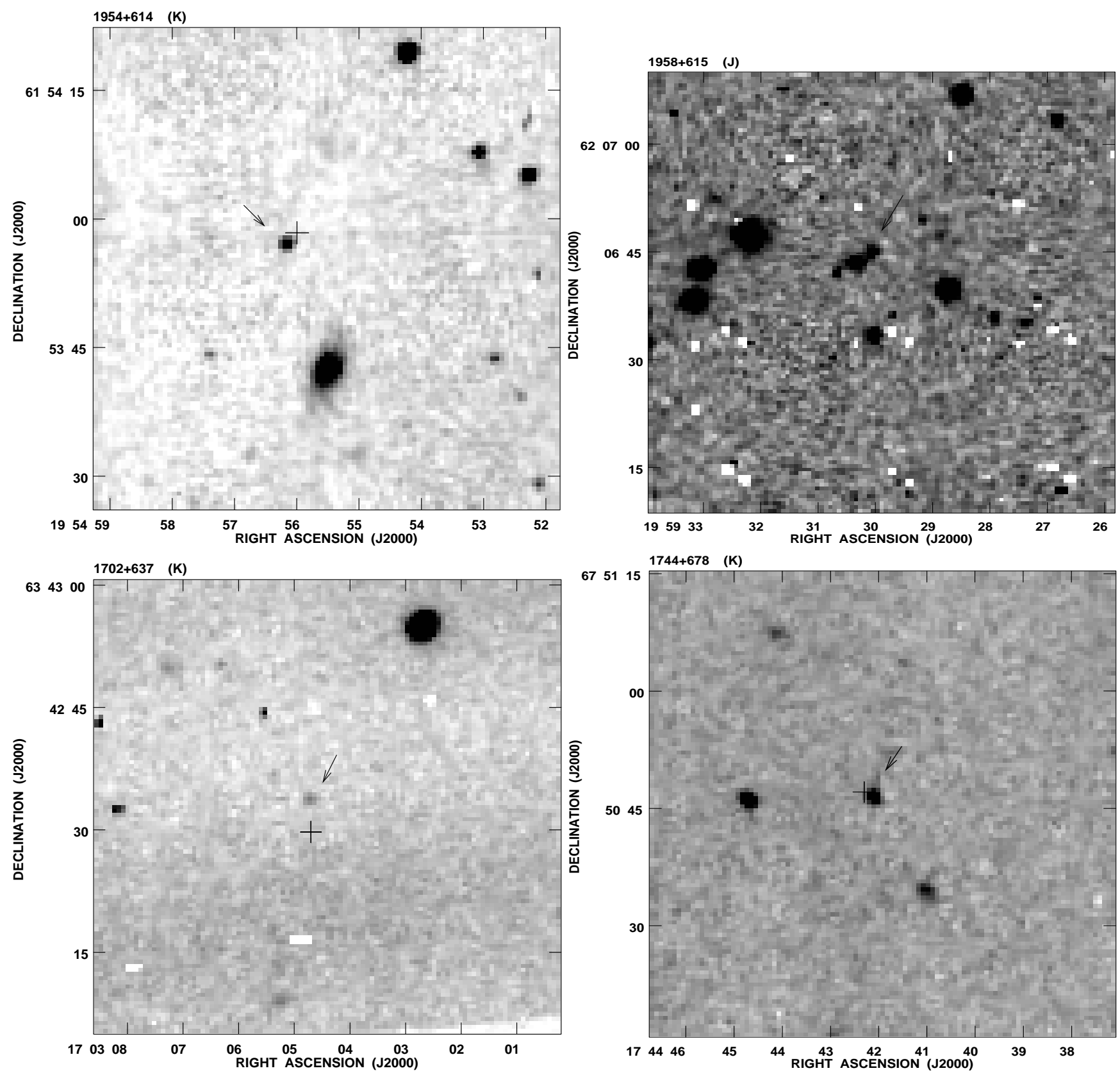

Fig. 1. continued

\section{IR imaging of the gravitational lens system $1600+434$}

One of the main objectives of the WENSS survey was to increase the small number of known gravitational lenses by mapping flat-spectrum radio sources, in order to provide for the first time a sample large enough to do reasonable statistics. In addition, optical/IR follow-up studies of confirmed lens systems allow the determination of the redshifts of the lensed objects and provide information about the lensing galaxy type. In our run of 1995 August, we performed IR imaging of the object $1600+434$, a lensed quasar at redshift 1.6, which consists of two images, separated by 1.4 arcsec, both in the radio and in the optical (Jackson et al. 1995). The lens has been detected in the $K$ - and $J$-bands but not resolved, although the seeing was good, probably because our pixel was 0.55 arcsec and undersampled the point spread function (PSF). In order to estimate the IR magnitudes and colours of the lensing galaxy, we have tried to carefully subtract the two quasar images using the astrometry of these two point sources from Jackson et al. (1995) and the astrometry of the lensing galaxy obtained from HST images (Jackson, private communication). We computed the pixel positions of the two quasar components assuming that the position of the lensing galaxy coincided with the light peak of the image. The quasars subtraction procedure was carried out 

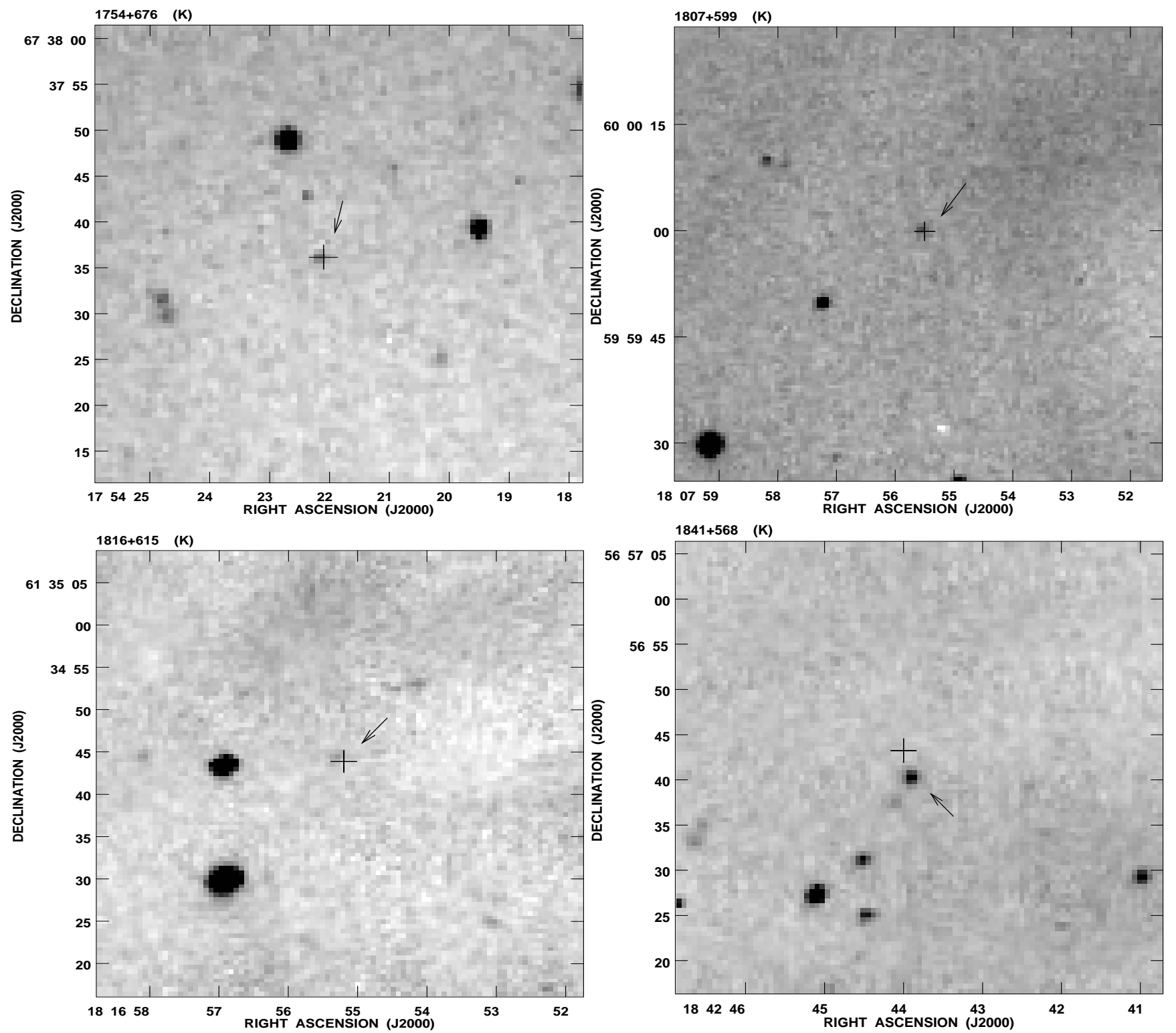

Fig. 1. continued

constructing two empirical two-dimensional PSFs. To this aim, after selecting some isolated stars in the field, we calculated the FWHM for all these objects and used its mean value to construct a gaussian PSF. The intensity of the PSF to be subtracted for the North Western image of the quasar has been obtained with a trial and error procedure which best eliminates the extension to the North West of our blended $J$ - and $K$-band images. The intensity of the PSF corresponding to the other quasar image was then fixed by the flux ratio in the radio (1.25) (Jackson, private communication). Figure 2 shows the $J$-band image of the lens system before and after subtraction of the two quasar images. This procedure allowed us to estimate the magnitude of the lensing galaxy in the two bands, which is listed in Table 6 together with the observing parameters.
We would like to stress that the photometric information on the lensing galaxy which we extract from our data is based on the VLA and HST astrometry and on the radio flux ratio for the two quasar images and is only partially affected by our relatively poor detector sampling. The stated accuracy in the lensing galaxy magnitude takes into account the uncertainty in the registration of the positions of the quasar images on the pixel grid.

\section{Conclusions}

We presented the results of deep IR imaging for three subsamples of radio sources of the WENSS catalogue: 36 USS, 14 GPS and 13 FS sources. We show images in the $J$ - or $K$-bands of the identified objects: 29 USS sources, 

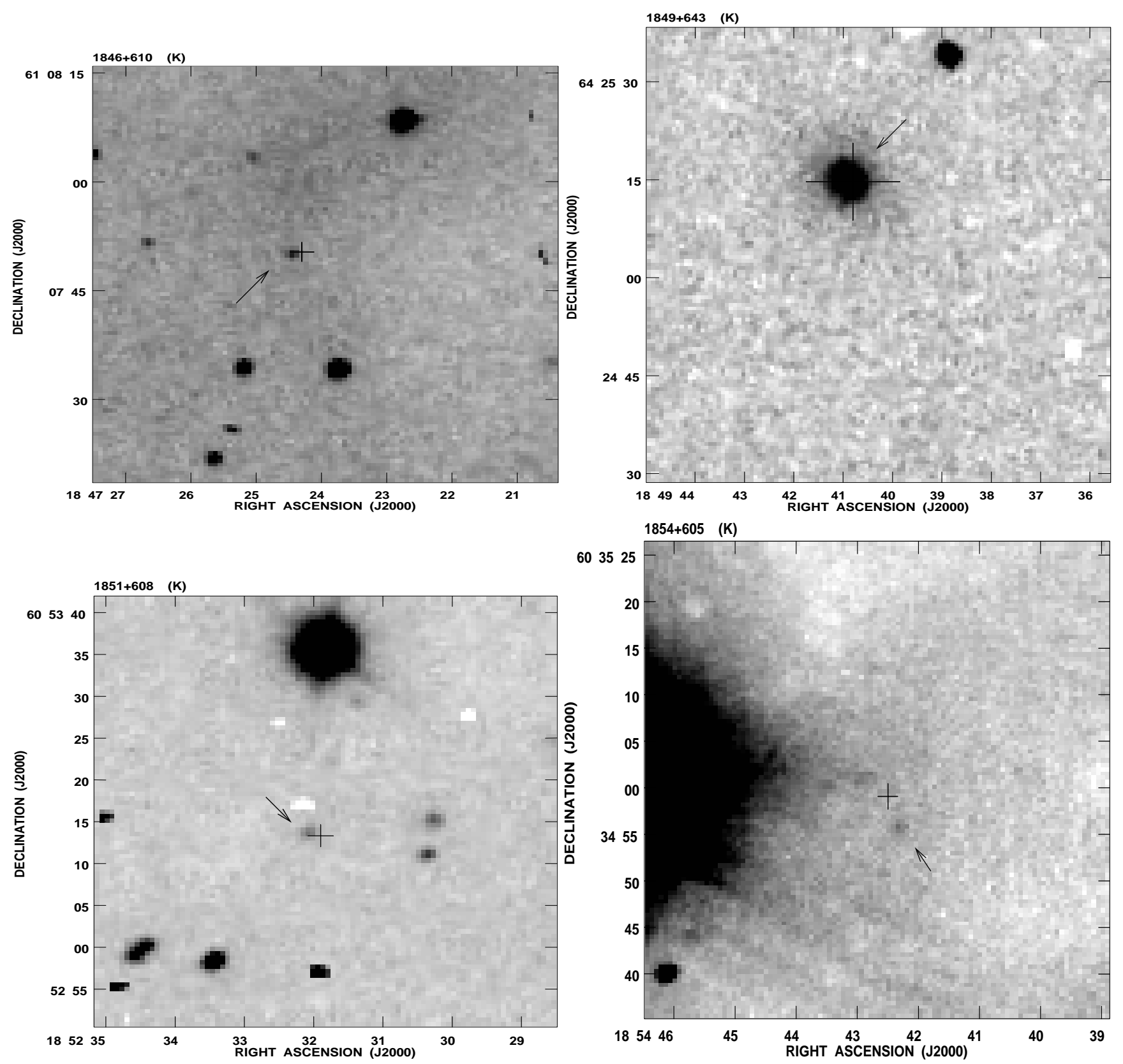

Fig. 1. continued

13 GPS sources and 10 FS sources. We have found IR counterparts for $44 \%$ of USS sources, $93 \%$ of GPS sources and $77 \%$ of FS sources. In addition, there are uncertain IR counterparts for $36 \%$ of USS sources and $15 \%$ of FS sources. In some cases, we find the IR counterparts of radio sources still lacking optical identifications. We remark that for the USS sources we find a larger percentage of certain IR identifications for the $\mathrm{A}$ and $\mathrm{B}$ sub-samples than for the high flux control sub-sample C.
We analyse the IR-radio alignment effect on 15 USS and 9 GPS radio galaxies and we do not find evidence for an alignment of the IR morphology with the radio axis.

Finally, we present the $J$-band image of the gravitational lens system $1600+434$, before and after subtraction of the quasar images. This procedure allows us to provide an estimate of the $J$ - and $K$-magnitudes of the lensing galaxy. 

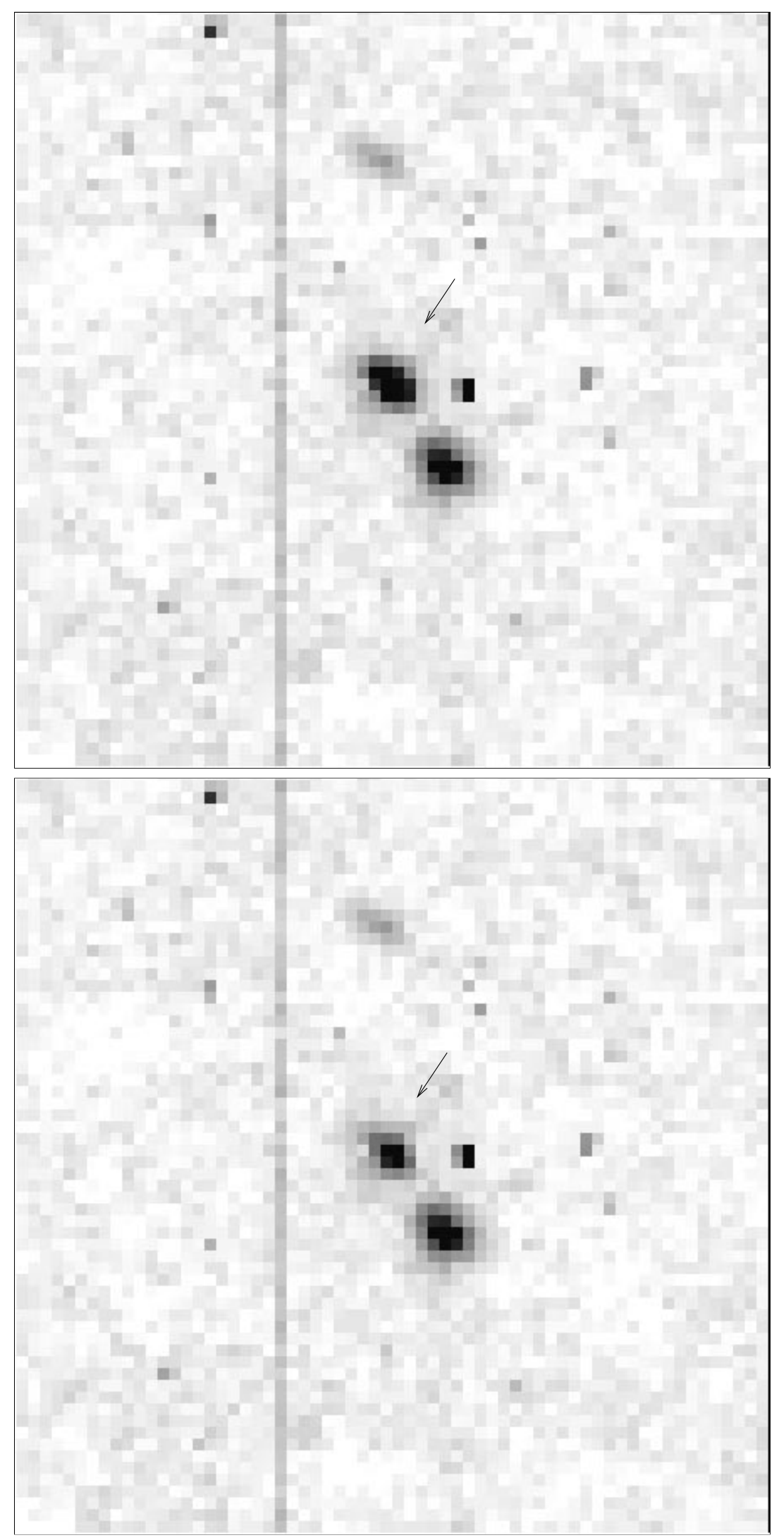

Fig. 2. The $J$-band image (North is to the top and East to the left) of the gravitational lens system $1600+434$, before a) and after b) the subtraction of the two quasar images. Of the two bright objects in the field the one to the north-west is the partial superposition of the two quasar images and of the lensing galaxy, while the one to the south-east is a nearby galaxy 
Acknowledgements. We thank Ruggero Stanga and Carlo Baffa for their support to the observations with ARNICA at the NOT (La Palma). We are grateful to Leslie Hunt for her kindness in helping with the data reduction, and to Richard Hook for providing the "drizzling" software. We thank George Miley for the hospitality at the Sterrewacht Observatory of Leiden and Malcolm Bremer, Andrea Cimatti, Roeland Rengelink and Ignas Snellen for useful discussions and for providing unpublished data.

\section{References}

Condon J.J, Broderick J., 1985, AJ 90, 2450

Dunlop J.S., Peacock J.A., 1993, MNRAS 263, 936

Gregory P.C., Condon J.J., 1991, ApJS 75, 1011
Hook R.N., Fruchter A.S., 1997, in: Astronomical Data Analysis Software and Systems VI, ASP Conf. Ser. 125, 147

Hunt L., et al., 1994, Arcetri Technical Report, No. 4

Hunt L., et al., 1998, AJ 115, 2594

Jackson N., et al., 1995, MNRAS 274, L25

Lilly S.J., Longair M.S., 1984, MNRAS 211, 833

Lisi F., et al., 1994, in: Proceedings of SPIE Technical Conference 1946. Orlando, p. 594

McCarthy P.J., 1993, ARA\&A 31, 693

Rengelink R.B., et al., 1997, A\&A 124, 259

Rigler M.A., et al., 1992, ApJ 385, 61

Snellen I.A.G., et al., 1996, MNRAS 279, 1294

Snellen I.A.G., et al., 1998, A\&AS 131, 435

Tyson J.A., 1986, J. Opt. Soc. Am. A 3(12), 2131 